\title{
Short Chain Acyl-Coenzyme A Dehydrogenase (SCAD) Deficiency Immunochemical Demonstration of Molecular Heterogeneity Due to Variant SCAD with Differing Stability
}

Etsuo Naito, Yasuhiro Indo, and Kay Tanaka

Yale University School of Medicine, Department of Human Genetics, New Haven, Connecticut 06510

\begin{abstract}
Using a $\left[{ }^{35} \mathrm{~S}\right]$ methionine labeling/immunoprecipitation technique, we have previously shown that cultured skin fibroblast from three patients with short chain acyl-CoA dehydrogenase (SCAD) deficiency each synthesize a normal-sized (41 kD) variant SCAD in an amount comparable to that of normal cells. In the current study, these same cell lines were reexamined with immunoblot analysis. In one cell line (YH2065) no SCAD protein was detectable. In the other two deficient cell lines, the amount of variant SCAD was similar to, or only slightly less than, normal. These results suggested that SCAD-YH2065 is labile. In the pulse-labeling experiments, labeled SCAD was readily detectable for at least $30 \mathrm{~h}$ in a normal control and two other SCAD-deficient cell lines. In contrast, the labeled SCAD band in YH2065 cells was barely detectable at $6 \mathrm{~h}$ and undetectable at $20 \mathrm{~h}$. $\left[{ }^{35} \mathrm{~S}\right]$ Methionine-labeling in the presence of rhodamine 6G demonstrated that SCAD-YH2065 was synthesized as a 44-kD precursor and imported normally into mitochondria, as were the normal SCAD and two other variant SCADs, excluding the possibility that SCAD-YH2065 is a truncated precursor that cannot be imported into mitochondria. These results indicate that the mutations responsible for SCAD deficiency are heterogeneous, and emphasize the importance of using both radiolabeling and immunoblotting when evaluating such genetic defects at the protein level.
\end{abstract}

\section{Introduction}

Hereditary short chain acyl-coenzyme A dehydrogenase (SCAD) ${ }^{1}$ deficiency is an inborn error of fatty acid metabolism that has recently been described in three infants $(1,2)$. The main clinical features in two of them were neonatal metabolic acidosis and urinary excretion of ethylmalonate. One of them died in the neonatal period (1). The third patient had a history of progressive skeletal muscle weakness, developmental delay, and muscle carnitine deficiency (2). Butyryl-CoA dehydro-

Address reprint requests to Dr. Kay Tanaka, Yale University School of Medicine, Department of Human Genetics, 333 Cedar Street, P.O. Box 3333, New Haven, CT 06510.

Received for publication 18 July 1989.

1. Abbreviation used in this paper: SCAD, short chain acyl-CoA dehydrogenase.

J. Clin. Invest.

(C) The American Society for Clinical Investigation, Inc. $0021-9738 / 89 / 11 / 1671 / 04 \$ 2.00$

Volume 84, November 1989, 1671-1674 genating activity in cultured fibroblasts from these three patients, assayed in the presence of anti-medium chain acyl-CoA dehydrogenase antibody, was found to be $<11 \%$ of controls, indicating a specific SCAD deficiency $(1,2)$.

SCAD (EC 1.3.99.2) is one of five enzymes belonging to the Acyl-CoA Dehydrogenase Family $(3,4)$. It catalyzes the first reaction in the $\beta$-oxidation of short chain fatty acids. Like other members of this gene family, SCAD is a tetrameric mitochondrial flavoprotein. Its subunit size is $41 \mathrm{kD}(5)$. It is encoded in the nucleus as a 44-kD precursor, transported into mitochondria, and proteolytically processed to the $41-\mathrm{kD}$ mature form (6). We have recently demonstrated, using labeling with ${ }^{35}$ S]methionine, immunoprecipitation, and electrophoretic analysis, that the cells from the three SCAD-deficient patients each produced an SCAD protein of normal size, suggesting that the biochemical defect in each of these patients is due to a point mutation in the SCAD gene (7). No genetic heterogeneity was detected. These results, however, did not exclude the possibility that more than one type of variant SCAD allele exists.

To further investigate this possibility, we studied variant SCAD in fibroblasts from the same three SCAD-deficient patients using immunoblot analysis and pulse labeling/chase experiments.

\section{Methods}

\section{Materials}

$\mathrm{L}-\left[{ }^{35} \mathrm{~S}\right]$ Methionine ( $>800 \mathrm{Ci} / \mathrm{mmol}$ ) was purchased from Amersham Corp. (Arlington Heights, IL). Formalin-fixed Staphylococcus aureus cells and ${ }^{14} \mathrm{C}$-labeled protein standard were obtained from Bethesda Research Laboratories (Gaithersburg, MD). Cell culture materials and rhodamine $6 \mathrm{G}$ were from Gibco Laboratories (Grand Island, NY) and Eastman Kodak Co. (Rochester, NY), respectively. The immunoblotting system was purchased from Bio-Rad Laboratories (Richmond, CA). The sources of three SCAD-deficient cell lines have been previously reported $(1,2)$. These cell lines are identified by our accession numbers as follows: YH2065 (L.N.) (1); YH1988 (A.A.) (1); and YH1950 (G-1) (2). Normal cell lines were obtained from the NIGMS Human Genetic Mutant Cell Repository (Camden, NJ).

\section{Procedures}

Purification of rat SCAD and the preparation of anti-rat SCAD antibody have previously been reported (5). Fibroblasts were grown in Eagle's MEM supplemented with 10\% FCS and kanamycin.

Immunoblot analysis. Immunoblot analysis was carried out as follows: The confluent monolayers of fibroblasts in two $150-\mathrm{cm}^{2}$ dishes were harvested by using a plastic scraper. Cells were washed twice with ice-cold PBS, and then stored at $-70^{\circ} \mathrm{C}$. The frozen pellets were resuspended in ice-cold lysis buffer $(0.5 \% \mathrm{NP}-40,50 \mathrm{mM}$ Tris- $\mathrm{HCl}, \mathrm{pH} 7.4$, $150 \mathrm{mM} \mathrm{NaCl}, 5 \mathrm{mM}$ EDTA, $0.02 \%$ sodium azide, and $1 \mathrm{mM}$ PMSF). The lysate was solubilized by boiling for $3 \mathrm{~min}$ with an equal volume of buffer containing $4 \%$ SDS, $125 \mathrm{mM}$ Tris- $\mathrm{HCl}, \mathrm{pH} \mathrm{6.8,20 \%}$ glycerol, 
$0.01 \%$ Bromophenol blue, and 10\% 2-mercaptoethanol. Samples (100 $\mu$ l) were electrophoresed in a $10 \%$ SDS-polyacrylamide gel with a $4 \%$ stacking gel. Proteins were then transferred from the polyacrylamide gel to a nitrocellulose membrane by overnight electroblotting. The transfer buffer was $25 \mathrm{mM}$ Tris-190 mM glycine, $\mathrm{pH}$ 8.3, diluted 4:1 with methanol. After the electrophoretic transfer, SCAD immunoreactive protein was detected using the Immuno-Blot alkaline phosphatase assay system (Bio-Rad Laboratories) as follows: The nitrocellulose membrane with immobilized proteins was soaked in blocking solution ( $150 \mathrm{mM} \mathrm{NaCl}, 10 \mathrm{mM}$ sodium phosphate buffer, $\mathrm{pH} 7.2$, containing $1 \%$ gelatin and $0.1 \%$ Tween 20 ) for $1 \mathrm{~h}$ at $40^{\circ} \mathrm{C}$. After rinsing with washing solution $(150 \mathrm{mM} \mathrm{NaCl}, 10 \mathrm{mM}$ sodium phosphate buffer, pH 7.2 , containing $0.25 \%$ gelatin and $0.1 \%$ Tween 20 ), the membrane was incubated for $1 \mathrm{~h}$ with a 1:300 dilution of anti-rat SCAD antibody. The membrane was washed, then incubated with a 1:3,000 dilution of goat anti-rabbit IgG alkaline phosphatase conjugate (Bio-Rad Laboratories). For color development of alkaline phosphatase, the nitrocellulose membrane was immersed in carbonate buffer $\left(0.1 \mathrm{M} \mathrm{NaHCO}_{3}\right.$, $1.0 \mathrm{mM} \mathrm{MgCl}_{2}, \mathrm{pH} 9.8$ ) containing 5-bromo-4-chloro-3-indolyl phosphate/nitro blue tetrazolium (Bio-Rad Laboratories).

Pulse-labeling of fibroblasts with $\left[{ }^{35} S\right]$ methionine and pulse-chase. Confluent monolayers of fibroblasts in two $25-\mathrm{cm}^{2}$ dishes were labeled with $\left[{ }^{35} \mathrm{~S}\right]$ methionine $(100 \mu \mathrm{Ci})$ in $2.5 \mathrm{ml}$ of labeling medium containing $60 \%$ Puck's saline G, $15 \%$ dialyzed FCS, and $10 \%$ glucose as previously described (8). The dishes were incubated at $37^{\circ} \mathrm{C}$ for $1 \mathrm{~h}$. After removing the labeling medium, cells were washed twice with PBS and then harvested by adding $1 \mathrm{ml}$ of NETS/methionine buffer $(150 \mathrm{mM}$ $\mathrm{NaCl}, 10$ mM EDTA, pH 7.4, 0.5\% Triton X-100, 0.25\% SDS, and 2\% unlabeled methionine) to each dish. When rhodamine $6 \mathrm{G}$ was used, it was added to the medium in a $75-\mathrm{cm}^{2}$ dish at a final concentration of $2.1 \mu \mathrm{M}, 30 \mathrm{~min}$ before the medium was replaced with the labeling medium containing $\left[{ }^{35} \mathrm{~S}\right]$ methionine. The dish was then incubated at $37^{\circ} \mathrm{C}$ for $1 \mathrm{~h}$ in the labeling medium containing the same concentration of rhodamine $6 \mathrm{G}$. In the chase experiments, the labeling of cells was performed as described above. After a 1-h pulse, the medium was replaced with growth medium containing no label, and the cells were harvested at 6,20 , and $30 \mathrm{~h}$, respectively. The labeled cells were rapidly washed twice with PBS, and then extracted as described above.

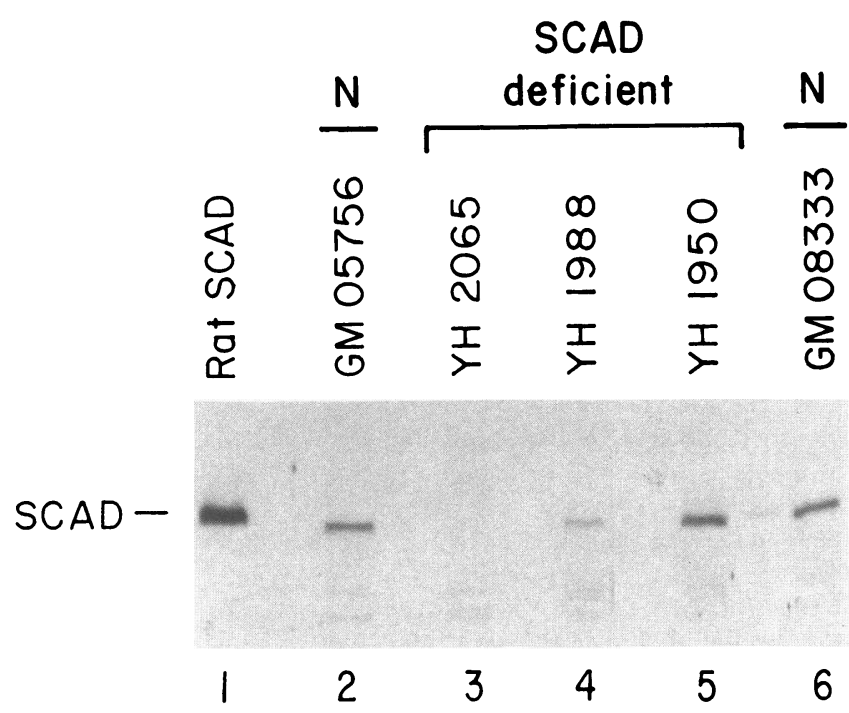

Figure 1. Immunoblot analysis of SCAD in extracts of normal and SCAD-deficient fibroblasts. Purified rat liver SCAD, 10 ng (lane 1 ) and human fibroblasts soluble protein, $100 \mu \mathrm{g}$ (lanes 2-6) were subjected to SDS-PAGE in a $10 \%$ polyacrylamide gel, then transferred to a nitrocellulose membrane and visualized as described in Methods. The extracts were from two control fibroblasts (lanes 2 and 6) and three SCAD-deficient lines (lanes 3-5) as indicated in the figure. The position of the SCAD protein is indicated on the left side.

\section{Results}

Immunoblot analysis. Fig. 1 shows the immunoblot of SDSPAGE of control purified rat liver SCAD and fibroblast extracts from two normal and three SCAD-deficient cell lines. The migration of pure rat SCAD was slightly slower (lane 1 ) than SCAD in the normal human fibroblast extracts (lanes 2 and 6). An SCAD of normal size was detected in the extract of YH1950 cells in an amount comparable to the normal controls (Fig. 1, lanes 2, 5, and 6). In contrast, no immunoreactive SCAD was detectable in the extract of YH2065 cells (lane 3). The immunoreactive SCAD in YH1988 cell was of normal size, but its amount is somewhat less than in the two normal and YH1950 lines.

Pulse-chase experiment. The absence of immunoreactive SCAD protein in YH2065, despite the ability of the cells to

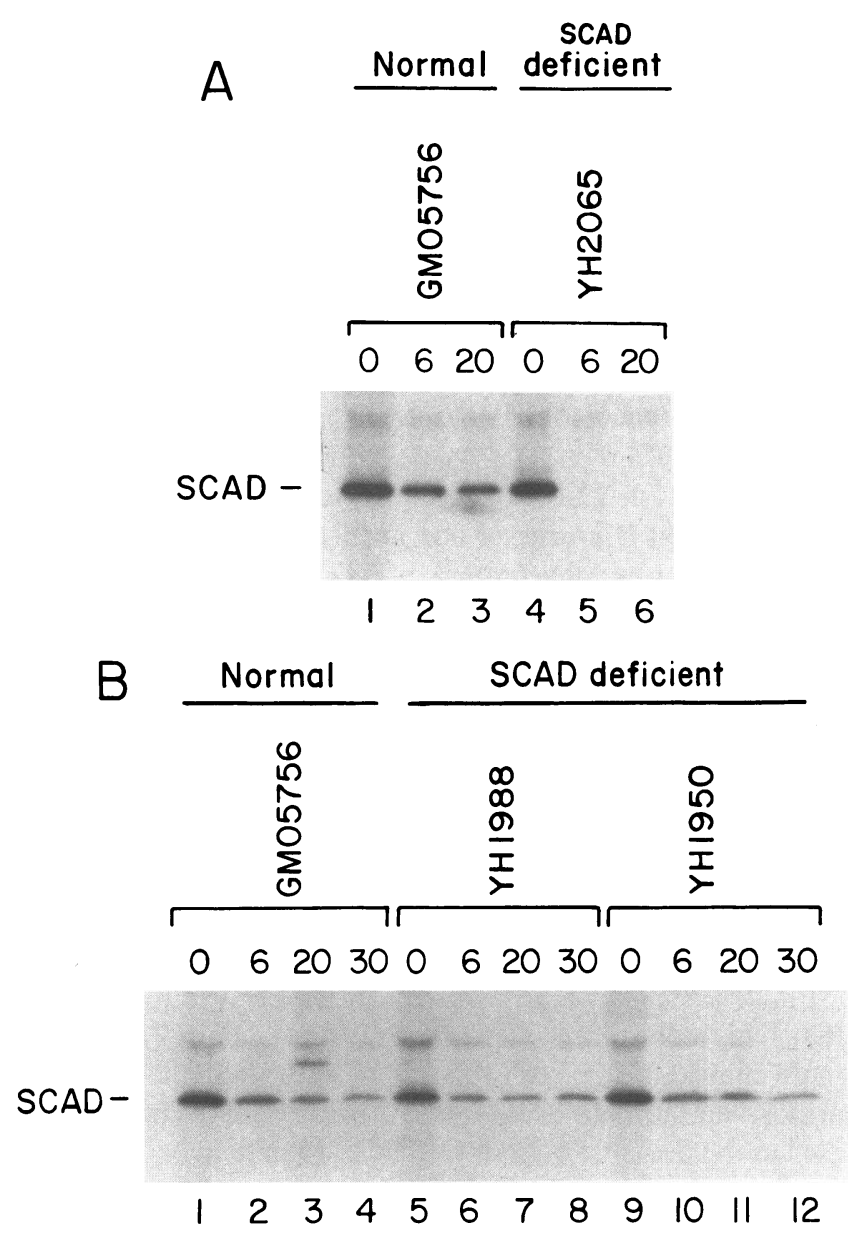

Figure 2. Pulse-chase experiments of SCAD in a normal and three SCAD-deficient fibroblast lines. Fibroblasts were grown in two $25-\mathrm{cm}^{2}$ dishes, and pulse labeling was done as described in Methods. $(A)$ After a 1-h pulse-labeling, the labeling medium with $\left[{ }^{35} S\right]-$ methionine was replaced with growth medium and the preparation chased for 6 and $20 \mathrm{~h}$, respectively. Lanes 1 and 4 , immediately after 1-h pulse; lanes 2 and 5, 6-h chase; lanes 3 and 6, 20-h chase. (B) After a $1-\mathrm{h}$ pulse-labeling, the labeling medium with $\left[{ }^{35} \mathrm{~S}\right]$ methionine was replaced with growth medium and the preparation chased for 6 , 20 , and $30 \mathrm{~h}$, respectively. Lanes 1,5 and 9 , immediately after $1-\mathrm{h}$ pulse-labeling; lanes 2, 6, and 10,6-h chase; lanes 3, 7, and 11, 20-h chase; lanes 4,8 , and 12,30 -h chase. Cell line numbers are shown at the top of each lane. The position of mature SCAD is indicated on the left side. 


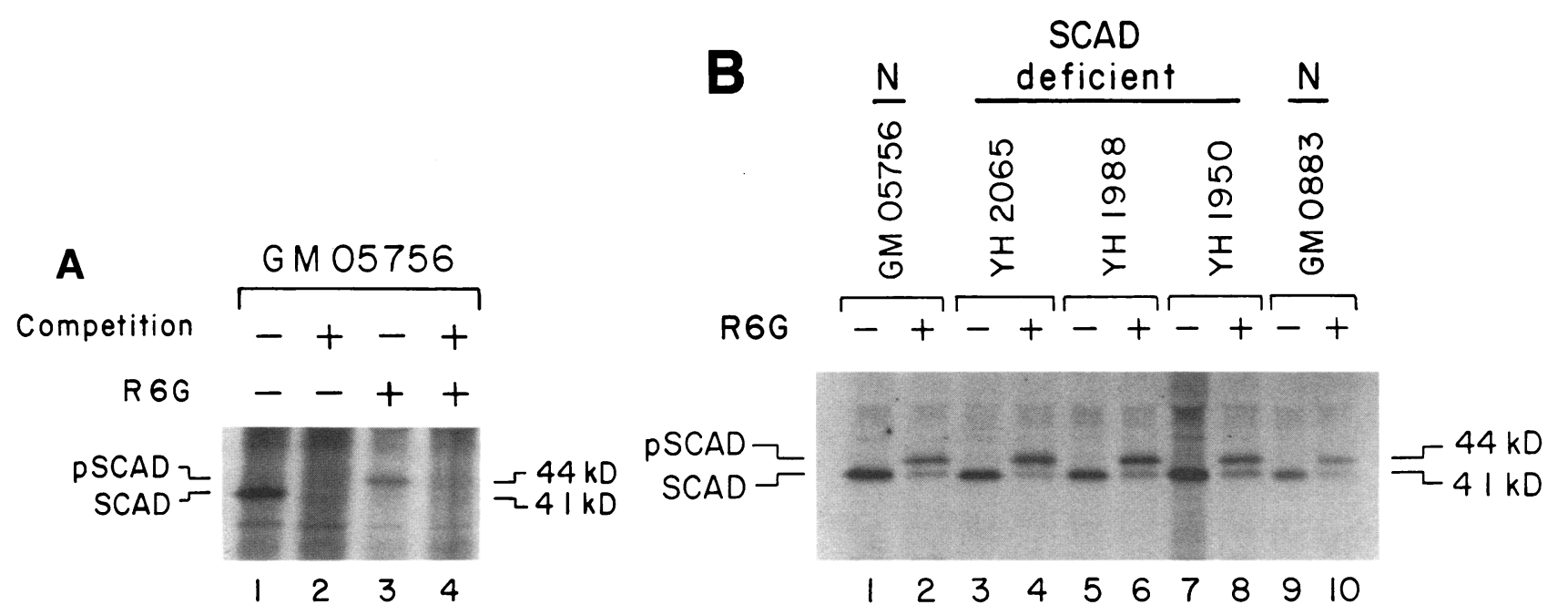

Figure 3. Identification of the precursor SCAD synthesized in a normal and three SCAD-deficient cell lines. The confluent monolayers in two $25-\mathrm{cm}^{2}$ dishes for mature SCAD or in one $75-\mathrm{cm}^{2}$ dish for precursor SCAD were labeled. After harvesting with NETS/methionine buffer, the cell extracts were combined, and the immunoprecipitates were analyzed on $10 \%$ SDS-PAGE as previously reported (8). $(A)$ Mature and precursor SCAD synthesized in normal cultured fibroblasts. (Lanes 1 and 2) Cells were labeled with $\left[{ }^{35}\right.$ S]methionine in the absence of rhodamine $6 \mathrm{G}$, and immunoprecipitated without (lane 1 ), and after addition of $7 \mu \mathrm{g}$ of pure rat SCAD (lane 2, competition experiment). (Lanes 3 and 4 ) Labeling was done in the presence of $2.1 \mu \mathrm{M}$ rhodamine $6 \mathrm{G}$, and immunoprecipitated without (lane 3 ) and after addition of $7 \mu \mathrm{g}$ of pure rat SCAD (lane 4, competition experiment). (B) Mature SCAD and its precursor synthesized in three SCAD-deficient cell lines. Lanes 3-8 each represent a SCAD-deficient cell line. Cell line numbers are shown at the top of each lane. Precursor and mature SCAD, and rhodamine $6 \mathrm{G}$ are abbreviated to pSCAD, SCAD, and R6G, respectively. Molecular sizes shown on the right are expressed in kilodaltons.

synthesize SCAD protein, suggested that variant SCADYH2065 might be very labile. In order to test this possibility, we pulse-labeled cells for $1 \mathrm{~h}$ with $\left.{ }^{35} \mathrm{~S}\right]$ methionine and then chased the labeled SCAD. The intensity of the fluorographic band for SCAD progressively decreased at 6 and $20 \mathrm{~h}$ in the normal cell line. However, the signal was quite strong at $20 \mathrm{~h}$ (Fig. $2 \mathrm{~A}$, lanes 1,2 , and 3 ) and still detectable at $30 \mathrm{~h}$ (Fig. $2 \mathrm{~B}$, lane 4). In YH1988 and 1950, the SCAD band was readily detectable up to $30 \mathrm{~h}$ as in the case of the normal cells (Fig. $2 B$, lanes 5-12). In line YH2065, immediately after the $1 \mathrm{~h}$ pulselabeling, a SCAD protein was synthesized in the amount comparable to that of the normal control and the two other SCAD-deficient cell lines (Fig. $2 A$, lane 4). However, it was barely detectable as early as $6 \mathrm{~h}$ after chase, and completely disappeared after a 20-h chase (Fig. $2 A$, lanes 5 and 6 ).

Electrophoretic analysis of SCAD labeled with $\left[{ }^{35} S\right]$ methionine. In order to study the synthesis of the SCAD precursor in normal and mutant cells, labeling was carried out with $\left[{ }^{35} \mathrm{~S}\right]-$ methionine in the presence of rhodamine $6 \mathrm{G}$. Rhodamine $6 \mathrm{G}$, an inhibitor of the mitochondrial energy metabolism, is known to inhibit the mitochondrial uptake of the precursor of nuclear encoded mitochondrial enzymes, leading to the suppression of their posttranslational processing $(6,9,10)$.

When normal cells were labeled in the absence of rhodamine $6 \mathrm{G}$, a sharp band with a molecular mass of $41 \mathrm{kD}$ was observed as previously reported (Fig. $3 A$, lane 1 ). When celllabeling was performed in the presence of rhodamine $6 \mathrm{G}$, the intensity of the $41-\mathrm{kD}$ band greatly decreased and a new $44-\mathrm{kD}$ band was detected (Fig. $3 A$, lane 3). Both 41- and 44-kD bands were not detectable when an excessive amount $(7 \mu \mathrm{g})$ of pure rat SCAD was added to each immunoprecipitation mixture before the addition of the antibody (Fig. $3 \mathrm{~A}$, lanes 2 and 4). This confirms that the 41- and $44-\mathrm{kD}$ bands are indeed the mature and precursor SCAD, respectively. When the SCAD synthesis was studied in the three SCAD-deficient cell lines using the same method, $41-$ and $44-\mathrm{kD}$ bands were detected in the absence and presence of rhodamine $6 \mathrm{G}$, respectively, as seen with normal cells (Fig. $3 B$, lanes 3-8). The intensity of both precursor and mature enzyme protein in the three SCAD-deficient cell lines was comparable to those in the normal cell line.

\section{Discussion}

We have previously shown that each of three SCAD-deficient cell lines can produce a $\left.{ }^{35} \mathrm{~S}\right]$ methionine-labeled SCAD of normal size. In this study, immunoblot analysis of the SCAD produced by these same cell lines has revealed that no immuno cross-reactive SCAD is detectable in one of the mutant cell lines (YH2065). This suggested that YH2065 cells synthesize a variant SCAD(s) at a rate comparable to that in normal cells and two other SCAD-deficient cell lines, but this variant SCAD is extremely labile. The variant SCAD in two other mutant lines appears as stable as, or slightly less stable than, normal SCAD. Pulse-labeling/chase experiments confirm that variant SCAD-YH2065 is indeed labile, while the stability of the variant SCAD in two other deficient cell lines is similar to their normal counterpart. We have previously shown that the half-life of the precursor in the cytosol is markedly shorter than that of the mature form in the mitochondria (6). However, all three variant SCADs are synthesized as a $44-\mathrm{kD}$ precursor, presumably imported into the mitochondria and processed to the mature $41-\mathrm{kD}$ form in a normal fashion. Thus, this excludes the possibility that the 41-kD SCAD produced in YH2065 represents a truncated precursor which cannot properly be imported into mitochondria, leading to degradation in the cytosol.

We have previously shown that normal amounts of appropriately sized $\mathrm{mRNA}$ are produced in all three SCAD-deficient cell lines (7). Taken in conjunction with these results, it seems 
likely that the SCAD deficiency in the three mutant cell lines studied here is probably due to a point mutation in each case. It is obvious, however, that at least the mutation in YH2065 is distinctly different from those in the other two cell lines, demonstrating molecular heterogeneity in the mutation of SCAD gene. This information will be useful for further pinpointing the mutation in each cell line using the polymerase chain reaction (11).

The results from the present study are also important, emphasizing that the two immunochemical methods used in these experiments examine somewhat different aspects of the protein under study. The $\left[{ }^{35} \mathrm{~S}\right]$ methionine-labeling/ immunoprecipitation technique reflects the cells' ability to synthesize protein, since an extremely small amount of newly synthesized protein can be detected due to the extremely high specific activity of $\left[{ }^{35} \mathrm{~S}\right]$ methionine. In contrast, immunoblot analysis represents the amount of protein present within the cells in the steady state. Most previous studies of proteins produced by mutant cell lines have been performed using only one of these methods. This can lead to an obvious wrong conclusion about the nature of such a mutation. For instance, if the variant SCAD in YH2065 had only been tested by immunoblot analysis, the complete absence of SCAD protein could be interpreted as being due to a gross deletion, a transcriptional mutant, or a frame-shift point mutation leading to a premature stop codon near the amino terminus. Thus, investigators must use both methods when studying such inborn errors of metabolism at the protein level.

\section{Acknowledgments}

Purification of rat short chain acyl-CoA dehydrogenase and preparation of anti-rat SCAD antibody was carried out by Dr. Yasuyuki Ikeda in this laboratory. Cultured skin fibroblasts from three patients with hereditary short chain acyl-CoA dehydrogenase deficiency were kindly provided by Drs. Carol Green, University of Colorado, Denver, CO; Vivian E. Shih, Massachusetts General Hospital, Boston, MA; and Susan Winters, Valley Children's Hospital, Fresno, CA. We thank Dr. Jerry Vockley for reading, and Mrs. Connie Woznick for preparing, this manuscript.

This work was supported by grants from the National Institutes of Health (DK38153) and March of Dimes (I-378).

\section{References}

1. Amendt, B. A., C. Greene, L. Sweetman, J. Cloherty, V. Shih, A. Moon, L. Teel, and W. J. Rhead. 1987. Short-chain acyl-coenzyme A dehydrogenase deficiency. Clinical and biochemical studies in two patients. J. Clin. Invest. 79:1303-1309.

2. Coates, P. M., D. E. Hale, G. Finocchiaro, K. Tanaka, and S. C. Winter. 1988. Genetic deficiency of short-chain acyl-coenzyme A dehydrogenase in cultured fibroblasts from a patient with muscle carnitine deficiency and severe skeletal muscle weakness. J. Clin. Invest. 81:171-175.

3. Matsubara, Y., Y. Indo, E. Naito, H. Ozasa, R. Glassberg, J. Vockley, Y. Ikeda, J. Kraus, and K. Tanaka. 1989. Molecular cloning and nucleotide sequence of cDNAs encoding the precursors of rat long chain acyl-coenzyme A, short chain acyl-coenzyme A and isovalerylcoenzyme A dehydrogenases: sequence homology of four enzymes of the acyl-CoA dehydrogenase family. J. Biol. Chem. 264:16321-16331.

4. Ikeda, Y., C. Dabrowski, and K. Tanaka. 1983. Separation and properties of five distinct acyl-CoA dehydrogenases from rat liver mitochondria: identification of a new 2-methyl branched chain acyl-CoA dehydrogenase. J. Biol. Chem. 258:1066-1076.

5. Ikeda, Y., K. O. Ikeda, and K. Tanaka. 1985. Purification and characterization of short-chain, medium-chain, and long-chain acylCoA dehydrogenases from rat liver mitochondria: isolation of the holo- and apoenzymes and conversion of the apoenzyme to the holoenzyme. J. Biol. Chem. 260:1311-1325.

6. Ikeda, Y., S. M. Keese, W. A. Fenton, and K. Tanaka. 1987. Biosynthesis of four rat liver mitochondrial acyl-CoA dehydrogenases: in vitro synthesis, import into mitochondria, and processing of their precursors in a cell-free system and in cultured cells. Arch. Biochem. Biophys. 252:662-674.

7. Naito, E., H. Ozasa, Y. Ikeda, and K. Tanaka. 1989. Molecular cloning and nucleotide sequence of complementary DNAs encoding human short chain acyl-coenzyme A dehydrogenase and the study of the molecular basis of human short chain acyl-coenzyme A dehydrogenase deficiency. J. Clin. Invest. 83:1605-1613.

8. Ikeda, Y., S. M. Keese, and K. Tanaka. 1985. Molecular heterogeneity of variant isovaleryl-CoA dehydrogenase from cultured isovaleric acidemia fibroblasts. Proc. Natl. Acad. Sci. USA. 82:7081-7085.

9. Morita, T., M. Mori, F. Ikeda, and M. Tatibana. 1982. Transport of carbamyl phosphate synthetase I and ornithine transcarbamylase into mitochondria. Inhibition by rhodamine 124 and accumulation of enzyme precursors in isolated hepatocytes. J. Biol. Chem. 257:1054710550.

10. Fenton, W. A., A. M. Hack, J. P. Kraus, and L. E. Rosenberg. 1987. Immunochemical studies of fibroblasts from patients with methylmalonyl-CoA mutase apoenzyme deficiency: detection of a mutation interfering with mitochondrial import. Proc. Natl. Acad. Sci. USA. 84:1421-1424.

11. Saiki, R. K., D. H. Gelfand, S. Stoffel, S. J. Scharf, R. Higuchi, G. T., Horn, K. B. Mullis, and H. A. Erlich. 1988. Primer-directed enzymatic amplification of DNA with a thermostable DNA polymerase. Science (Wash. DC). 239:481-487. 